J3eA, Journal sur l'enseignement des sciences et technologies de l'information et des systèmes, Volume 2, Hors-Série 2, 6 (2003)

DOI : http://dx.doi.org/10.1051/bib-j3ea:2003606

(C) EDP Sciences, 2003

Des mini-convertisseurs électromécaniques au service de nouvelles fonctionnalités

B. Nogarede

Groupe « Machines et Mécanismes Electroactifs - EM²»

LEEI - UMR CNRS 5828

INPT / ENSEEIHT

2 rue Charles Camichel

B.P. 7122

F-31071 Toulouse Cedex 7, France

Bertrand.Nogaredelleei.enseeiht.fr 


\title{
Des mini-convertisseurs électromécaniques au service de nouvelles fonctionnalités.
}

\author{
Bertrand NOGAREDE \\ Groupe « Machines et Mécanismes Electroactifs - EM² » \\ LEEI - UMR CNRS n ${ }^{\circ} 5828$ - INPT / ENSEEIHT \\ 2, rue Charles Camichel - B.P. 7122 - 31071 TOULOUSE Cedex 7 \\ E-mail :. Bertrand.Nogarededleei.enseeiht.fr
}

\begin{abstract}
Résumé
Après un bref récapitulatif des divers procédés physiques exploitables en conversion électromécanique de l'énergie, le présent article propose un tour d'horizon des enjeux modernes de l'électromécanique dans la gamme des actionneurs de dimensions centi- ou déci-métriques. Le potentiel des nouvelles technologies considérées est évalué au travers de différents exemples d'actionneurs innovants visant à répondre à l'augmentation des performances ou à l'extension des fonctionnalités requises face à divers types d'application.
\end{abstract}

\section{Introduction}

Les applications modernes de l'électromécanique se caractérisent par une intégration de plus en plus poussée de fonctions d'actionneur et de capteur au sein de mécanismes alliant hautes performances massiques et fonctionnalités évoluées. Cette tendance est d'autant plus marquée dans le domaine des actionneurs de dimensions centi- ou décimétriques qu'elle ouvre la voie à une nouvelle génération de dispositifs électromécaniques aptes à relever le défi du « tout électrique » (ou tout au moins du « plus électrique »), dans des domaines aussi variés que l'automobile (système de contrôle-moteur, motorisation de confort...), le génie biomédical (prothèses et orthèses motorisées, microchirurgie robotisée...), la domotique (automatismes domestiques), ou encore l'aéronautique et l'espace (actionneurs de commande de vol, de type EHA ou EMA, gestion des circuits de carburant...). Les progrès récemment accomplis dans le domaine des matériaux, qu'ils soient «passifs" (matériaux magnétiques composites) ou «électroactifs» (céramiques piézoélectriques ou électrostrictives, massives, multicouches, ou composites, alliages magnétostrictifs, alliages à mémoire de forme...) laissent aujourd'hui apparaître un champ d'innovation très prometteur, en vue d'aboutir à des dispositifs à hautes performances massiques bénéficiant en outre d'un haut niveau d'intégration fonctionnelle. Le présent article propose un tour d'horizon non exhaustif des nouveaux enjeux de l'électromécanique face à divers types d'applications émergeantes. Après une brève analyse des différentes filières technologiques en présence, les exemples plus particulièrement considérés mettent tour à tour l'accent sur la nécessité d'une d'intégration fonctionnelle de plus en plus poussée, la recherche de performances sensiblement améliorées en termes d'efforts massiques, la mise au point de dispositifs capables de contrôler simultanément plusieurs degrés de liberté, et enfin le potentiel que représente l'idée d'un actionnement « réparti » pour des applications telles que le contrôle électroactif de la turbulence en aéronautique.

\section{Une grande diversité d'effets électromécaniques exploitables}

$\mathrm{Au}$ cours de la dernière décennie, les procédés exploitables en conversion électromécanique de l'énergie se sont considérablement élargis grâce notamment à l'avènement des matériaux «électroactifs ». Dans le domaine dimensionnel considéré, les concepts d'actionneurs qui en résultent constituent une véritable rupture technologique laissant entrevoir de nouvelles possibilités, tant sur le plan fonctionnel que du point de vue des performances de la conversion d'énergie réalisée [1]. Soulignons que ce potentiel tient essentiellement à la possibilité de générer des efforts spécifiques élevés (contraintes «motrices» de l'ordre de $40 \mathrm{MPa}$ dans les céramiques piézoélectriques PZT ou de l'ordre de $100 \mathrm{Mpa}$ dans les alliages à mémoire de forme) dans un encombrement réduit (la conversion d'énergie s'opère dans le volume même du matériaux), tandis que les procédés « classiquement» utilisés, fondés sur l'interaction «à distance » de corps magnétiques, conduisent à des pressions équivalentes d'entrefer inférieures, par principe, à $1 \mathrm{MPa}$ (dispositif non-supraconducteurs). Certes, l'amplitude des déplacements élémentaires produits à l'aide des transducteurs électroactifs reste limitée (déformation relative de l'ordre de 1000 parties par million pour les céramiques piézoélectriques multicouches et de 8000 ppm dans le cas des alliages à mémoire de forme). Néanmoins, la possibilité de cumuler ces micro-déplacements dans l'espace (par exemple en utilisant la flexion d'une poutre allongée) ou dans le temps (grâce à la transmission, par contact intermittent, d'un mouvement vibratoire à haute fréquence) donne lieu à des performances remarquables en termes de puissance spécifique. Ainsi, comme l'illustre la Figure 1, la comparaison des performances intrinsèques associés aux principaux procédés exploitables démontre clairement le potentiel que représente l'exploitation de ces nouveaux matériaux. 


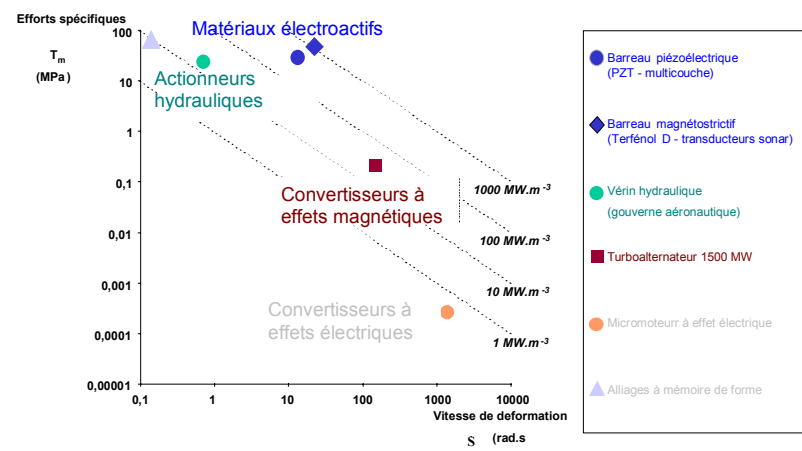

Figure 1. Comparaison des effets électromécaniques exploitables en termes d'énergie spécifique [2]

Certes, il faut noter que, menée à l'échelle des principes et non des structures de convertisseurs, cette comparaison ne tient pas compte des « infrastructures » nécessaires à la mise en œuvre des effets électromécaniques considérés (culasses magnétiques, éléments de guidage mécanique, systèmes de refroidissement...). Ainsi, les niveaux de puissance spécifique indiqués ne sauraient être confondus avec les performances effectives associés à des objets électromécaniques opérationnels. Néanmoins, ces valeurs limites restent globalement cohérentes avec les tendances observées à l'échelle d'actionneurs de référence : en termes d'effort massique par exemple, un actionneur piézoélectrique rotatif de dimension centimétrique (couple massique de l'ordre de $10 \mathrm{~N} . \mathrm{m} . \mathrm{Kg}^{-1}$ ) est globalement dix fois plus performant qu'un actionneur à aimants permanents de même couple nominal.

\section{Vers une intégration fonctionnelle poussée}

Les applications modernes de l'électromécanique, notamment dans le domaine des servocommandes ou des dispositifs d'assistance électrique, se caractérisent souvent par la nécessité d'intégrer des fonctions d'actionneur et de réducteur mécaniques, éventuellement associés à un frein d'immobilisation. Afin de réduire l'encombrement global $\mathrm{du}$ dispositif, on peut chercher à intégrer les fonctions motrices et réductrices au sein d'une même structure magnétique, comme l'illustre le concept de moto-réducteur présenté sur la Figure 2. Basé sur une architecture de machine synchrone à aimants permanents, cet actionneur présente la particularité de comporter un rotor excentré roulant à l'intérieur du stator (via une piste dentée) qui décrit dès lors un mouvement hypocycloïdal [3]. Outre ses avantages en termes d'intégration fonctionnelle, ce principe tire directement profit des efforts radiaux qui s'exercent sur le rotor, efforts dont le travail est nul dans les structures à rotor centré, bien que leur intensité soit généralement supérieure aux efforts tangentiels générés. Ainsi, au delà du prototype de validation évoqué ici (structure bipolaire tétraphasée dont le rapport d'engrenage est de 3), l'optimisation du concept vise à l'heure actuelle à augmenter sensiblement (de l'ordre de $50 \%$ ) le couple massique de la structure préconisée.

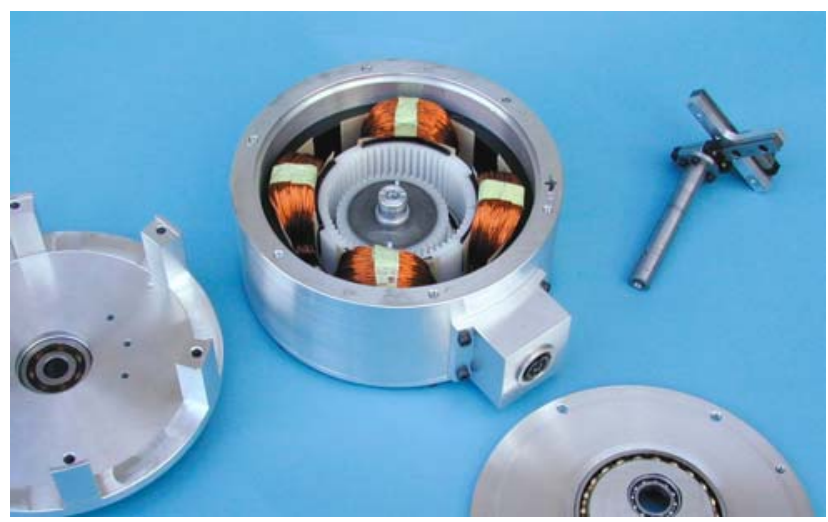

Figure 2. Prototype de machine à aimants permanents à réducteur hypocycloïdal intégré [3]

Outre la mise au point de moto-réducteurs intégrés, l'exploitation de nouveaux concepts de conversion permet également d'envisager des structures motrices optimisées à l'échelle même du mécanisme dans lequel elles interviennent. Ainsi, le mécanisme présenté sur la Figure 3, qui correspond à la partie active d'une prothèse de la main motorisée à deux degrés de mobilité (flexion de poignet, préhension), met clairement en évidence le potentiel que représente la technologie des moteurs piézoélectriques pour ce type d'application [4] : deux fonctions motrices peuvent être logées à la place du mécanisme classique à un seul degré de liberté actionné par un micromoteur à courant continu (pour une masse globalement réduite), tandis que la discrétion acoustique, la précision de positionnement et le freinage à manque de courant caractérisant l'entraînement par moteurs piézoélectriques (ici deux moteurs à rotation de mode) constituent autant d'avantages à considérer.

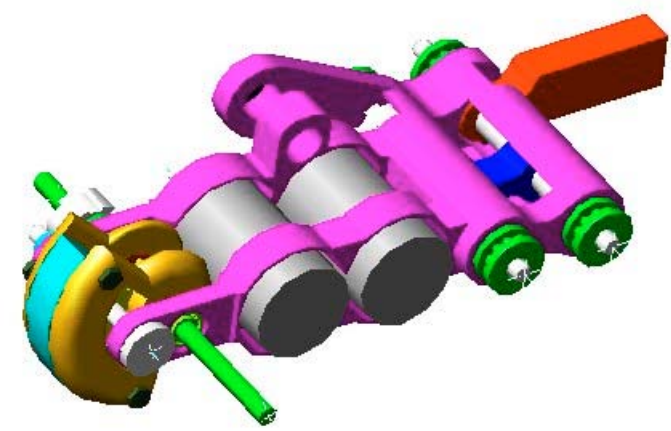

Figure 3. Mécanisme de prothèse de la main à deux degrés de mobilité à motorisation piézoélectrique [4]

\section{Efforts élevés à basse vitesse et entraînement « direct »}

Parallèlement à la démarche conduisant à mieux intégrer les fonctions de réduction dans les mécanismes à motorisation électrique, l'émergence de nouveaux besoins visant à satisfaire des spécifications de type effort élevé à basse vitesse, sous des contraintes de masse et d'encombrement de plus en plus sévères (notamment dans le domaine aéronautique et spatial), favorise le développement de concepts d'actionneurs assurant un entraînement « direct» de la charge mécanique (absence d'étages de réduction dans la chaîne cinématique). Le mode de transmission «par contact» utilisé dans les moteurs 
piézoélectriques constitue de ce point de vue un atout réel dans la mesure où les efforts surfaciques mis en jeu à l'interface stator-rotor (de l'ordre de $4 \mathrm{MPa}$ ) sont très supérieurs aux pressions d'entrefer équivalentes couramment exploitées dans les mini-machines à effet magnétique (de l'ordre de 0,1 Mpa). Soulignons en outre que ces niveaux de pression, essentiellement limités par des considérations d'ordre tribologique (compromis performance - durée de vie), restent encore très inférieures aux contraintes motrices potentiellement exploitables dans le matériau électroactif (C.f. §2). La Figure 4 illustre ces possibilités au travers d'un nouveau concept de piézoactionneur rotatif opérant en mode quasi-statique (stator à pince déformées à l'aide de céramiques PZT multicouches), destiné à l'entraînement direct d'une vanne de carburant aéronautique et dimensionné pour développer un couple maximum de 10 N.m (vitesse maximale de l'ordre de 5 tr. $\mathrm{min}^{-1}$ ), pour une masse totale de $1,5 \mathrm{Kg}$.

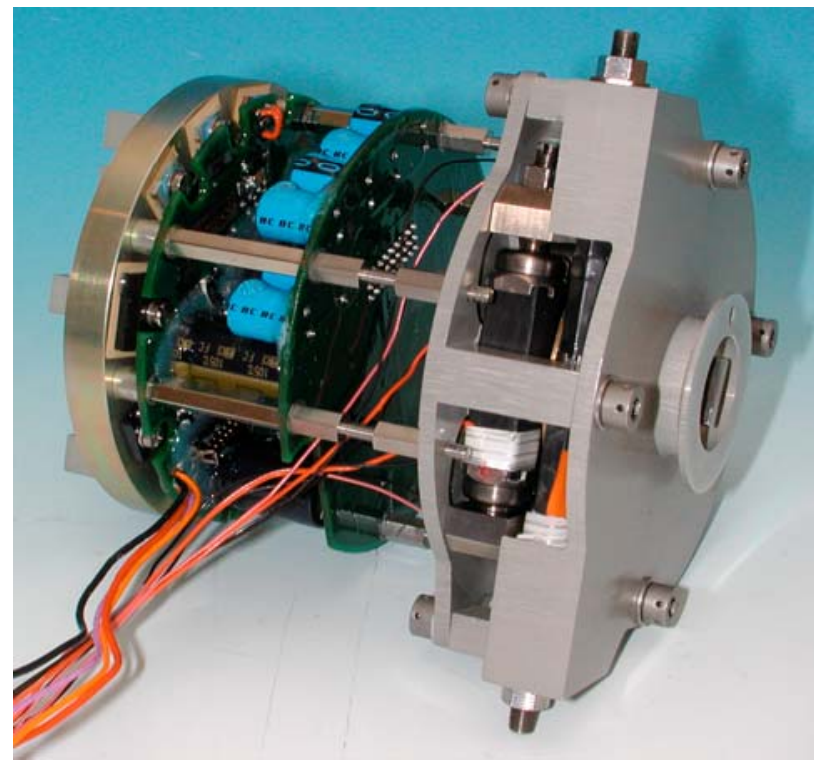

Figure 4. Actionneur piézoélectrique à déformation « quasi-statique » associé à son électronique de pilotage

\section{Des actionneurs combinant plusieurs degrés de liberté}

Les nouvelles fonctionnalités résultant de la gestion simultanée de plusieurs degrés de liberté au sein d'un même mécanisme (micro-chirurgie robotisée, micropositionnement pour la micro-électronique ou la microscopie en champ proche [5] par exemple) constituent également pour le chercheur un champ d'investigation particulièrement riche. A l'opposé de l'approche visant à combiner les différents mouvements requis au sein de mécanismes multi-axes relativement sophistiqués, il s'agit plutôt ici d'imaginer des structures d'actionneur intrinsèquement conçus pour gérer conjointement les différents déplacements requis. Citons à titre d'exemple le cas de l'actionneur piézoélectrique plan schématisé sur la Figure 6 dont le principe de fonctionnement repose sur l'excitation monophasée d'une onde de flexion stationnaire. Le réglage de la fréquence d'alimentation permet alors de contrôler la trajectoire du mobile sur un plan. Le démonstrateur réalisé, de dimension centimétrique, s'avère relativement intéressant du point de vue de ses performances dans la mesure où il permet de déplacer une masse de $2 \mathrm{Kg}$ à une vitesse de l'ordre de $10 \mathrm{~mm} . \mathrm{s}^{-1}[6]$.
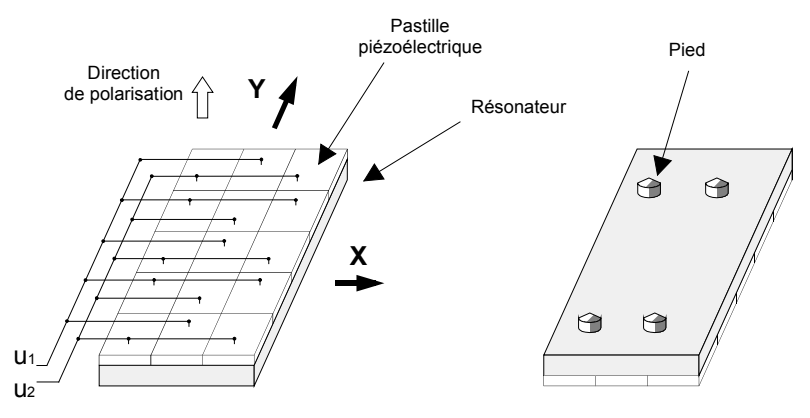

vue de dessus

vue de dessous
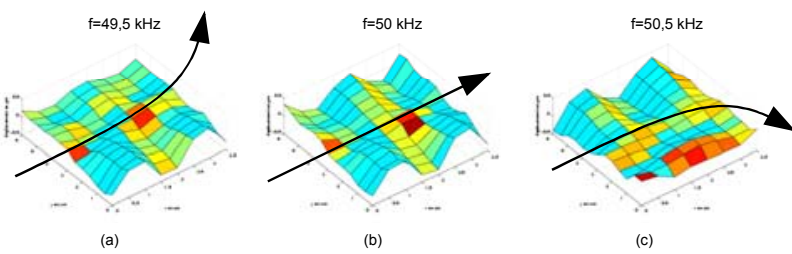

Figure 5. Actionneur piézoélectrique plan - pilotage en ligne droite (b), en virage à gauche (a) et à droite (c) [6]

\section{A la recherche de fonctionnalités inédites : actionneurs répartis pour le contrôle électroactifs d'écoulements en aéronautique}

Si, d'un point de vue fonctionnel, l'actionnement électromécanique est généralement abordé à l'échelle d'un système à constantes localisées au sein duquel interagissent des sous-ensembles électriques et mécaniques interconnectés par un ou plusieurs convertisseurs électromécaniques, le développement récent de fonctions à caractère "distribué » (contrôle actif de vibration, réduction de bruit, contrôle de forme...) ouvre la voie à de nouveaux concepts d'actionneurs dont les parties actives sont susceptibles de "fusionner» avec l'architecture dont ils assurent le contrôle. Le domaine émergeant du contrôle électroactif d'écoulements constitue un exemple particulièrement révélateur de cette tendance. Parmi les différents sujets qui intéressent ce nouveau champ d'investigation, le contrôle dynamique de la turbulence de paroi au moyen d'ondes de surface excitées le long du profil est susceptible de tirer avantageusement profit des nouvelles possibilité offertes par les matériaux électroactifs (utilisées sous forme «massive» ou « composites»). Soulignons que ces investigations, dont l'objectif est à terme de réduire la traînée de frottement associée par exemple à l'écoulement de l'air le long d'une voilure aéronautique, demeurent au stade actuel des connaissances une problématique relativement «amont» dont il s'agit pour l'instant de démontrer la faisabilité. La Figure 7 présente ainsi un dispositif d'étude à caractère expérimental développé en vue de vérifier l'effet d'ondes de déformation transversales (par rapport à la direction de l'écoulement) sur les stries de proche paroi qui peuplent la couche limite et qui jouent un rôle majeur dans le phénomène de turbulence [7]. Signalons que ce dispositif à échelle réduite 
(expérimentation prévues dans un premier temps sous faibles vitesses d'écoulement) permet de contrôler des déplacements en bout de lames de l'ordre de $3 \mathrm{~mm}$, la bande passante du système étant limitée à $150 \mathrm{~Hz}$. Soulignons qu'une configuration à échelle unitaire (vitesses d'écoulements sub- voire super-soniques) conduirait à un système d'actionneurs réparti pouvant être intégré dans la peau du profil étudié dans la mesure où l'amplitude des déplacements requis serait alors inférieure au millimétrique.

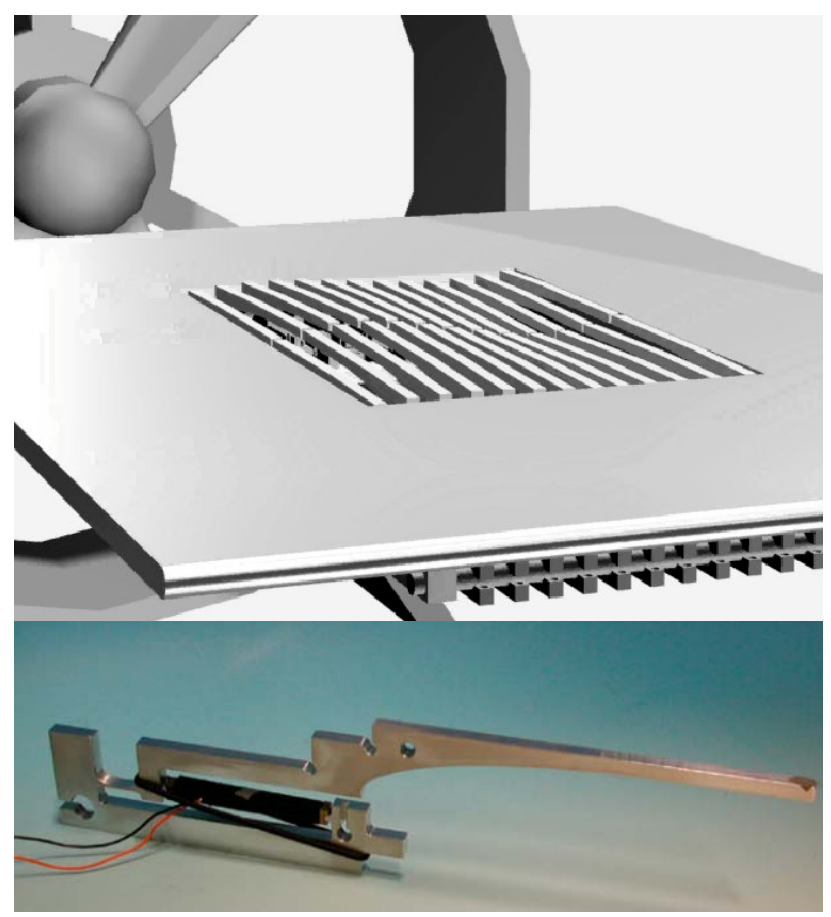

Figure 7. Actionneur piézoélectrique à lames multiples pour la réduction de traînée : le concept PALM [7]

\section{Conclusions}

Le domaine de la conversion électromécanique de l'énergie constitue de toute évidence un champ d'investigation riche et prometteur, notamment dans le domaine des actionneurs de dimensions centi- ou décimétriques.

Le champ des procédés physiques exploitables dans ce domaine s'est d'une part sensiblement élargi, grâce notamment au développement des matériaux « électroactifs » dont les performances, les fonctionnalités et la souplesse de mise en œuvre ouvrent la voie à de nouveaux concepts de convertisseurs. Exploitée au sein de moteurs ou d'actionneurs, cette technologie permet d'envisager des gains substantiels en termes d'effort massique, grâce à un entraînement «direct" (à basse vitesse) de la partie mobile. Certes, un certain nombre de difficultés freinent encore le développement de cette famille d'actionneurs, en raison notamment du problème encore mal maîtrisé que constitue l'optimisation des conditions de contact à l'interface stator-rotor. Néanmoins, les résultats obtenus en recherche dans le domaine témoignent du potentiel que représente cette technologie dès lors qu'il s'agit, par exemple, de contrôler un déplacement selon plusieurs degrés de liberté, en mettant en jeu un nombre réduit de pièces mécaniques et un système d'alimentation et de commande relativement simple.
Par ailleurs, il faut noter que cette démarche d'innovation technologique est favorisée par la puissante motivation que constitue l'émergence de nouvelles problématiques en matière d'application de l'électromécanique. Ainsi, la nécessité croissante de contrôler des processus physiques au caractère de plus en plus «distribué » invite naturellement le chercheur à imaginer des dispositifs à motricité répartie, tirant directement profit des possibilités d'intégration structurelles qu'offrent par principe les matériaux électroactifs.

Enfin, pour être menés de manière sûre et efficace, les développements technologiques envisagés doivent nécessairement s'appuyer sur une réflexion méthodologique appropriée. Cet effort vise, d'une part, à développer des modèles véritablement prédictifs, en particulier dans le domaine des matériaux électroactifs excités sous forts signaux. D'autre part, le caractère relativement innovant des objets considérés limite de toute évidence le recul empirique dont peut disposer le concepteur, en particulier lors des étapes de dimensionnement. Le recours à des procédures mathématiques adaptées, permettant par exemple de formuler et résoudre le problème en termes de « conception optimale », pourra alors avantageusement renforcer la portée opérationnelle des nouveaux concepts mis en avant.

\section{Références}

[1] T. Sashida , T. Kenjo, « An introduction to ultrasonic motors », Clarendon Press, OXFORD, 1993.

[2] B. Nogarede, "Machines électriques: conversion électromécanique de l'énergie », Traité de Génie Electrique, Techniques de l'Ingénieur, D3410 (2000).

[3] C. Henaux, G. Pons, B. Nogarede, «A novel type of permanent magnet actuator: the HYPOMAG structure», ICEM'2000, Espoo (Finland), 28-30 August, 2000.

[4] B. Nogarede, C. Henaux, J.-F. Rouchon, F. Léonard, R. Briot, L. Petit, P. Gonnard, B. Lemaire-Semail, F. Giraud, Ph. Kapsa, "Matériaux électroactifs et génie biomédical : étude d'une prothèse de la main actionnée par une motorisation piézoélectrique ", MGE'2000, Lille, 13-14 décembre 2000.

[5] N. Bonnail, D. Tonneau, H. Dallaporta, G.-A. Capolino, «Dynamic response of a piezoelectric actuator at low excitation level in the nanometer range», ICEM'2000, Espoo (Finland), 28-30 August, 2000.

[6] F. Galiano, B. Nogarede, «Un nouveau concept d'actionneur piézoélectrique plan monophasé à onde stationnaire », Revue Internalionale de Génie Electrique, Vol. 2, No/Ref. 4/1999.

[7] P. Konieczny, A. Bottaro, V. Monturet, B. Nogarede, « Active control of near-wall coherent structures », FEDSM'2002, Joint US ASME-European Fluids Engineering Summer Conference Montreal, Quebec (Canada), July 14-18, 2002. 